\title{
Direct Correction of Residual Symmetric Aberrations in Electron Holograms of Weak Phase Objects
}

\author{
Felix Kern ${ }^{1 *}$, Martin Linck ${ }^{2}$, Daniel Wolf ${ }^{1}$, Tore Niermann ${ }^{3}$, Himani Arora ${ }^{4}$, Nasim Alem ${ }^{5}$, Artur Erbe ${ }^{4}$, \\ Sibylle Gemming ${ }^{4}$ and Axel Lubk ${ }^{1}$ \\ 1. Leibniz Institute for Solid State and Materials Research, Institute for Solid State Research, 01069 \\ Dresden, Germany. \\ 2. CEOS GmbH, 69126 Heidelberg, Germany. \\ 3. Technische Universität Berlin, Institut für Optik und Atomare Physik, \\ 10623 Berlin, Germany. \\ 4. Helmholtz-Zentrum Dresden Rossendorf, 01328 Dresden, Germany. \\ 5. Department of Materials Science and Engineering, The Pennsylvania State University, University \\ Park, PA 16802, United States \\ * Corresponding author: f.l.kern@ifw-dresden.de
}

Thin TEM specimen are regarded as weak phase objects (WPOs), if the amplitude variation of the electron wave by the specimen can be neglected and the phase modulation is very small $(\ll \pi)$. Large classes of topical materials can be described in this approximation, such as most 2D materials, organic semiconductor materials or biological specimen. Due to the lack of amplitude (and hence intensity) contrast, conventional TEM (CTEM) investigations on WPOs are commonly performed under a certain defocus, which transfers part of the phase information to the recorded intensity. This intermixing contrast transfer from amplitude to phase and vice versa is commonly described by the phase contrast transfer function (PCTF), while the non-mixing contrast transfer for amplitude and phase is referred to as amplitude contrast transfer function (ACTF). Due to the transfer gap in the PCTF, the CTEM contrast transfer at low spatial frequencies is degraded in defocused images of WPOs (Fig. 1). By employing electron holography, however, both amplitude and phase of the electron wave can be reconstructed without a transfer gap. Having the whole wave information also enables the a-posteriori correction of geometric aberrations as it was already proposed in D. Gabor's seminal paper from 1948 [1]. The realization of his idea, however, remains challenging in the absence of additional knowledge about the sample, due to the lack of a criterion for a successful aberration correction.

Here we present a novel method for the a-posteriori correction of symmetric geometric aberrations in electron holograms, which analytically exploits the constant amplitude in images of WPOs and does not need any further knowledge about the object. The correction of residual aberrations results in a constant contrast transfer over a large band that is only decreases at higher spatial frequencies due incoherent aberrations (Fig. 1). This technique is demonstrated on electron holograms of multilayered boron nitride (h-BN), acquired at the Cc-corrected TEAM 1 microscope [2], where it is facilitating quantitative atomic-resolution imaging (Fig. 2). These aberrations are partially set deliberately (e.g., defocus) to select a proper region of interest on the sample as they lead to transfer of phase information to the amplitude (Fig. 2a) but also to a damping of higher spatial frequencies in the reconstructed phase (Fig. 2b). The corrected phase (Fig. 2d) exhibits atomic resolution in the multi-layered parts while preserving large range phase modulations stemming from the different projected potentials of different numbers of h-BN monolayers. The corrected (Fig. 2c) amplitude shows almost no amplitude variations proofing the validity of our approach. The ease of use of the proposed methods facilitates a flexible application to a wide range of WPOs including dose sensitive ones, benefitting from the "dose-free" aberration 
correction.

References:

[1] D Gabor, Nature 161 (1948), p. 777-778

[2] M Linck, P Ercius, C Ophus, N Alem, A Zettl and U Dahmen, Microscopy and Microanalysis 19, p. 1222-1223.

[3] We acknowledge funding from the European Research Council (ERC) under the Horizon 2020 research and innovation program of the European Union (grant agreement No 715620).

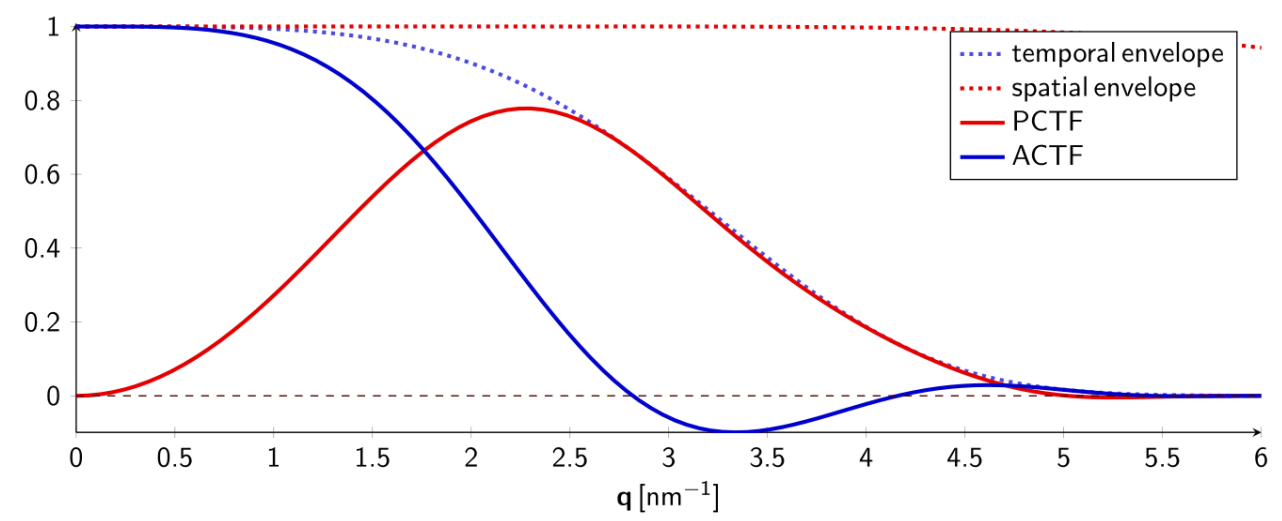

Figure 1. Phase and amplitude transfer functions as well as temporal and spatial envelope for typical high-resolution microscope parameters: a defocus of $11 \mathrm{~nm}$, a spherical aberration coefficient of $-10 \mu \mathrm{m}$, an acceleration voltage of $80 \mathrm{kV}$, a linear chromatic aberration coefficient of $1.41 \mathrm{~mm}$, a semi convergence angle of $0.15 \mathrm{mrad}$ and an energy width of $0.7 \mathrm{eV}$ (FWHM).
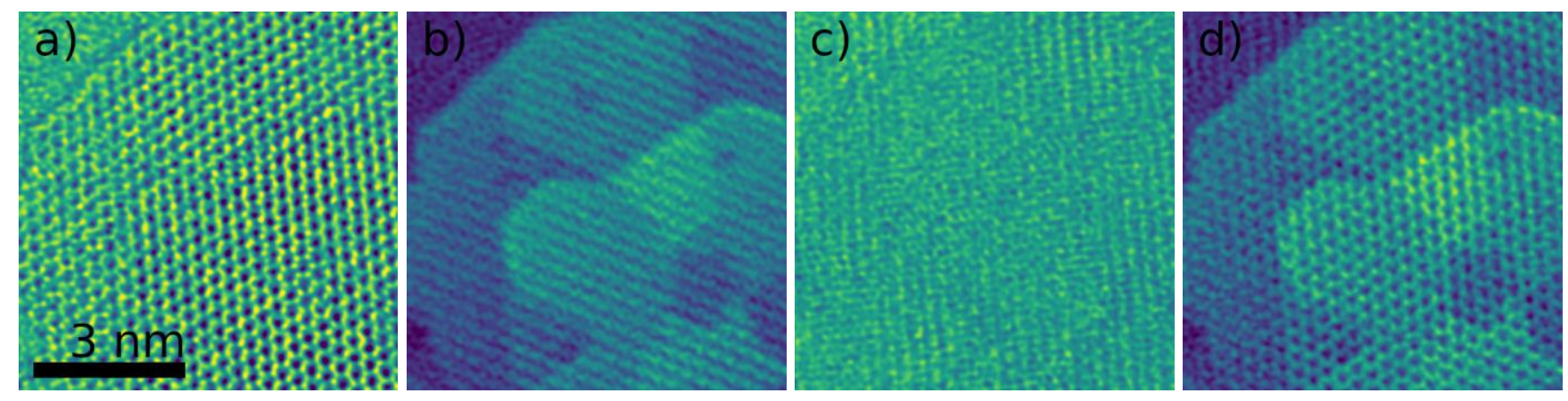

Figure 2. Reconstructed phase (a) and amplitude (b) of boron nitride multilayers. The amplitude contrast can be explained as transferred phase information in the weak phase object approximation. Amplitude (c) and phase (d) corrected with the presented method. The corrected amplitude shows just marginal modulation, the corrected phase contains atomically resolved information as well as low spatial frequency features. 